further studies to determine to what extent the quality of life in the city, as opposed to selective migration, can account for the enormous rural-urban differences in psychiatric morbidity. Replication of our study in other cities in Pakistan would be very useful.

However, the truly remarkable finding is not the prevalence of common mental disorders in urban Rawalpindi, which is more in line with rates reported elsewhere in the world. It is the exceptionally high rate of psychiatric morbidity in rural villages in Pakistan, recently confirmed by other investigators in another village near Gujar Khan (Hussain et al, 2000); this cries out for further research.

Regarding the use of male doctors to conduct the second-stage interviews of female subjects, we were obliged to do the same in the previous study in rural Chitral (Mumford et al, 1996). However we have found no psychometric inconsistencies between the three epidemiological surveys (in Chitral, Gujar Khan and Rawalpindi) to suggest that this was a source of bias while making psychiatric diagnoses according to ICD-10 criteria for research.

Hussain, N., Creed, F. \& Tomenson, B. (2000) Depression and social stress in Pakistan. Psychological Medicine, 30, 395-402.

Mumford, D. B., Nazir, M., Jilani, F. M. et al (1996) Stress and psychiatric disorder in the Hindu Kush. A community survey of mountain villages in Chitral, Pakistan. British Journal of Psychiatry, 168, 299-307.

_, Saeed, K., Ahmad, l., et al (1997) Stress and psychiatric disorder in rural Punjab. A community survey. British Journal of Psychiatry, I70, 473-478.

D. B. Mumford Division of Psychiatry, University of Bristol, 4I St Michael's Hill, Bristol BS2 8DZ

\section{Prevalence of depression in old age}

Korten \& Henderson (2000) described findings from a 1997 national survey, in which a "household sample of 10641 individuals representative of the adult population of Australia" was interviewed. They reported that "the prevalence of a diagnosis of any ICD-10 anxiety or depressive disorder ... declines for both men and women after the age of 55 years", and noted a trend for psychological symptoms to be fewer among the older age groups. Before accepting the findings as evidence that depression is less prevalent in old age (a conclusion that might affect decisions about allocation of resources), the following points should be noted (see Snowdon et al, 1998).
First, the (approximately) 1600 subjects aged over 65 years were not truly representative of the older population. The survey excluded the $10 \%$ of older Australians who were temporarily or permanently residing in institutes (e.g. hospitals, nursing homes, boarding houses), or homeless at the time of the survey. It also excluded those with moderate or severe dementia (Mini-Mental State Examination score $<18$ ). The prevalence of depression is considerably higher among those with physical disability, those in residential care and those with dementia.

Second, the instrument forming the core of the interview was the automated version of the Composite International Diagnostic Interview (CIDI), which discounts symptoms attributable to physical illness (Jorm, 2000). Studies that rely on subject-reported symptoms may underestimate the severity of depression in old age, since older patients with depression are less likely than younger patients to acknowledge having affective symptoms (Lyness et al, 1995).

Third, the response rate in this survey was $78 \%$, but the response rate of different age-groups was not known. In other surveys (e.g. Kramer et al, 1985), older subjects have been twice as likely as younger adults to decline involvement. Refusers are more likely to be depressed.

Finally, the report did not differentiate prevalence rates in 'young-old' and 'oldold' individuals, yet various researchers have found a progressive increase in rate from 55 to 85 years. Jorm (2000) commented on the lack of consistency between researchers regarding whether or not depression becomes less prevalent in old age.

Jorm, A. F. (2000) Does old age reduce the risk of anxiety and depression? A review of epidemiological studies across the adult life span. Psychological Medicine, 30, $11-22$

Korten, A. \& Henderson, S. (2000) The Australian National Survey of Mental Health and Well-Being. Common psychological symptoms and disablement. British Journal of Psychiatry, 177, 325-330.

Kramer, M., German, P. S., Anthony, J. C., et al (1985) Patterns of mental disorders among the elderly residents of Eastern Baltimore. Journal of the American Geriatrics Society, 33, 236-245.

Lyness, J. M., Cox, C., Curry, J., et al (1995) Older age and the underreporting of depressive symptoms. Journal of the American Geriatrics Society, 43, 216-221.

Snowdon, J., Draper, B., Chiu, E., et al (1998) Surveys of mental health and wellbeing: critical comments. Australasian Psychiatry, 6, 246-247.

J. Snowdon Department of Psychologica Medicine, University of Sydney, Rozelle Hospital, Rozelle, NSW 2039, Australia
Authors'reply: Professor Snowdon questions the validity of the results on the elderly from the Australian National Survey of Mental Health and Well-Being. He rightly points out that the survey failed adequately to cover the population living in institutional care, which was $9 \%$ of Australians over 65 in 1998. This is clearly acknowledged in earlier publications. Indeed, the indigenous people of Australia, people in prison, the homeless, the armed forces and the migrant population were also not included in numbers large enough to give stable prevalence estimates, mainly for the sake of economy in what was already a very large undertaking. We used "an unweighted sample with no group represented in a proportion greater than its frequency in the population" (Henderson et al, 2000).

The lack of information concerning the $22 \%$ non-responders is indeed regrettable, but does not detract from the finding, consistent with many of the studies cited in Jorm (2000), that the community-dwelling elderly displayed significantly lower levels of depressive symptomatology than younger cohorts. This was reflected in the prevalence rates and in all the scales of psychological distress measured in the survey: the 12-item General Health Questionnaire (GHQ-12), the 12-item Short-Form General Health Survey (SF-12), the Kessler-10 scale, the CIDI screen items for depression and finally the neuroticism items from the Eysenck personality questionnaire considered to reflect vulnerability to psychological symptoms. Each of these scales handles symptoms associated with physical disability in a different way. In all cases, the lower levels of symptomatology observed among 65to 70 -year-olds were maintained into the oldest age group (75 years and above), although the pattern is less stable than for younger age groups because of smaller numbers. The interested reader is referred to Jorm (2000) for a discussion of the possible mechanisms involved.

Information on mental disorders among the oldest old and institutional elderly are of crucial importance for advocacy. But this needs to be addressed in ways other than in large community surveys. This was made explicit from the beginning, where we stated that information on "some of the most significant elements in our society" would need special studies (Henderson et al, 2000). Any concern that our findings might affect decisions about allocation of resources is unlikely to be justified, because it assumes that administrators and policy-makers will 
make the grave error of extrapolating from community estimates to the special population of the elderly in hostels and nursing homes.

\section{Henderson, S., Andrews, G. \& Hall, W. (2000)}

Australia's mental health: an overview of the Genera

Population Survey. Australian and New Zealand Journal of Psychiatry, 34, 197-205.

Jorm, A. F. (2000) Does old age reduce the risk of anxiety and depression? A review of epidemiological studies across the adult life span. Psychological Medicine, 30, $11-22$.

A. Korten, S. Henderson Centre for Menta Health Research, The Australian National University, Canberra, ACT 0200, Australia

\section{Antidepressants and suicide risk}

Donovan et al (2000) make interesting points about deliberate self-harm (DSH) and antidepressant drugs, but their report as written is open to grave misinterpretation. Indeed, a reporter brought the article to my attention wanting to know why selective serotonin reuptake inhibitors (SSRIs) increased suicide risk relative to tricyclic antidepressants (TCAs).

A key problem with this cross-sectional, naturalistic study of DSH and antidepressant medications at emergency department presentation is that patients were not diagnosed. The authors write as if antidepressant medications are almost invariably prescribed to treat depression, yet clearly this is not always true. Even within mood disorders, patients may differ greatly in suicide risk. The authors found fragmentary evidence that patients on SSRIs may have been relatively treatment-resistant.

Moreover, SSRIs are prescribed for a growing spectrum of psychiatric illnesses beyond depression. The authors hint at the multiplicity of indications, mentioning enuresis as an indication (presumably for TCAs). Astoundingly, however, they never mention borderline personality disorder (BPD). Patients with BPD, known for their frequent parasuicidal gestures (Davis et al, 1999), are more likely to receive SSRIs than TCAs: partly because of their safety in overdose, partly for their benefit for impulsivity independent of mood disorder. Hence BPD and other patients at higher risk for DSH may have received SSRIs rather than TCAs. The authors mention this briefly ("... the question of whether patients prescribed TCAs were similar in terms of DSH risk to those prescribed SSRIs", p. 553) but fail to emphasise how crucial this issue is (Neither do they mention substance misuse, a further risk factor for self-destructive behaviour.) Given this likely diagnostic and prescriptive imbalance, it is unsurprising that more suicidal patients presenting at emergency departments were taking SSRIs.

In summary, without knowing that equivalent patient populations were receiving the two classes of medications, we cannot compare their effect on suicide risk.

Davis, T., Gunderson, J. G. \& Myers, M. (1999)

Borderline personality disorder. In The Harvard Medical School Guide to Suicide Assessment and Intervention (ed. D. G. Jacobs), pp. 3II-33I. San Francisco, CA: JosseyBass.

Donovan, S., Clayton, A., Beeharry, M., et al (2000) Deliberate self-harm and antidepressant drugs. Investigation of a possible link. British Journal of Psychiatry, 177, 55I-556.

J.C. Markowitz Weill Medical College of Cornell University, New York Presbyterian Hospital, Payne Whitney Clinic, 525 East 68th Street, Room 1322 , New York, NY I002I, USA

\section{Cognitive therapy and social functioning in chronic depression}

We clinicians constantly encounter patients with major depression in partial remission. They are no longer acutely depressed but continue to present with substantial functional impairment (Paykel et al, 1995). For treatment-resistant depression, only one pharmacological intervention can be recommended today with reasonable evidence, namely lithium augmentation (Austin et al, 1991; Aronson et al, 1996), but this may not be the answer for those with low-grade residual depression.

Scott et al (2000) demonstrated that cognitive therapy can help these people. Critically appraising their article in our evidence-based psychiatry case conference, however, it was very difficult for us to appreciate the substantive significance of this improvement, because only means and standard deviations of scores on the Social Adjustment Scale were reported. Analyses based on these data can show whether or not the treatment is better than the control condition, but cannot show how much better it is - a crucial piece of information for both patients and clinicians. We therefore resorted to the normative data for this scale (Bothwell \& Weissman, 1977).
Calculation based on the means and standard deviations under the assumption of a normal distribution showed that, at week 20,68\% of patients with residual depression reached the $95 \%$ range of the control subjects when treated with clinical management plus cognitive therapy, whereas only $45 \%$ did so when treated with clinical management only. This translates into a 'number needed to treat' of 4.4 (95\% CI 2.6-12.6).

This is an impressive figure. By adding 16 sessions of cognitive therapy to usual care, we can achieve social remission in one additional patient out of four, compared with continued standard care only. The original authors had concluded, "In patients showing only partial response to antidepressants, the addition of CT produced modest improvement in social and psychological functioning". We find that the improvement was more than modest and would be clinically meaningful.

Aronson, R., Offman, H. J., Joffe, R.T., et al (1996) Triiodothyronine augmentation in the treatment of refractory depression: a meta-analysis. Archives of General Psychiatry, 53, 842-848.

Austin, M.-P. V., Souza, F. G. M. \& Goodwin, G. M. (1991) Lithium augmentation in antidepressant-resistant patients. A quantitative analysis. British Journal of Psychiatry, 159, 510-514.

Bothwell, S. \& Weissman, M. M. (1977) Social impairments four years after an acute depressive episode. American Journal of Orthopsychiatry, 47, 23I-237.

Paykel, E. S., Ramana, R., Cooper, Z., et al (1995) Residual symptoms after partial remission: an important outcome in depression. Psychological Medicine, 25, II7I1180.

Scott, J., Teasdale, J. D., Paykel, E. S., et al (2000) Effects of cognitive therapy on psychological symptoms and social functioning in residual depression. British Journal of Psychiatry, I77, 440-446.

\section{S. Ito, H. Takeuchi, T. A. Furukawa}

Department of Psychiatry, Nagoya City University Medical School, Mizuho-cho, Mizuho-ku, Nagoya 467-860I, Japan

Author's reply: I am a strong advocate of the use of cognitive therapy in chronic and residual depressive disorders. I am therefore the last to disagree with the comments of Ito and colleagues that there is real benefit in providing psychosocial treatments to individuals with residual depressive symptoms. My comment on social functioning was not meant to underestimate the benefits, but paid heed to two factors. First, although individuals who received cognitive therapy undoubtedly 